\title{
C-terminal binding proteins: emerging roles in cell survival and tumorigenesis
}

\author{
Lee M. Bergman and Jeremy P. Blaydes \\ Cancer Sciences Division, School of Medicine, \\ University of Southampton, \\ U.K.
}

Correspondence to Lee M. Bergman, Somers Cancer Research Building, MP 824, Southampton General Hospital, Southampton SO16 6YD, U.K. Phone: +44 (0)23 8079 6531, Fax: +44 (0)23 8079 5152, email: L.M.Bergman@soton.ac.uk

Abbreviated title: CtBP proteins

Acknowledgements: supported by a grant (\# 2003: 713) from the U.K. Breast Cancer Campaign 
Within a cell, the levels and activity of multiple pro- and anti-apoptotic molecules act in concert to regulate commitment to apoptosis. Whilst the balance between survival and death can be tipped by the effects of single molecules, cellular apoptosis control pathways very often incorporate key transcription factors that co-ordinately regulate the expression of multiple apoptosis control genes. Cterminal binding proteins (CtBPs), which were originally identified through their binding to the Adenovirus E1A oncoprotein, have been described as such transcriptional regulators of the apoptosis program. Specifically, CtBPs function as transcriptional co-repressors, and have been demonstrated to promote cell survival by suppressing the expression of several pro-apoptotic genes. In this review we summarise the evidence supporting a key role for CtBP proteins in cell survival. We also describe the known mechanisms of transcriptional control by CtBPs, and review the multiplicity of intracellular signaling and transcriptional control pathways with which they are known to be involved. Finally we consider these findings in the context of additional known roles of CtBP molecules, and the potential implications that this combined knowledge may have for our comprehension of diseases of cell survival, notably cancer.

Keywords: CtBP, apoptosis, transcriptional repression, Golgi, cancer. 


\section{Discovery of CtBPs: cellular binding partners of transforming viral oncoproteins}

Isolation of the cellular binding partners of the viral transforming oncoproteins has, historically, proven to be a fruitful approach for the identification of key cellular regulators of proliferation and survival. E1A proteins of type $2 / 5$ adenoviruses, in particular, associate with multiple cellular proteins in order to reprogram cellular growth, survival and differentiation pathways to facilitate viral gene expression and replication. ${ }^{1,2}$ E1A proteins are encoded by two exons; the first exon, which contains conserved regions that bind to the cellular proteins $\mathrm{Rb}$ and $\mathrm{p} 300 / \mathrm{CBP}$, is sufficient for E1A to co-operate with activated ras in baby rat kidney (BRK) cell cotransformation assays. ${ }^{3,4}$ E1A truncation mutants lacking exon 2 are markedly more effective than wild type E1A in these assays, although intriguingly, the loss of these C-terminal sequences renders E1A unable to cooperate with Adenovirus E1B in transformation assays, or immortalize BRK cells in the absence of a co-operating oncogene..$^{3,5-7}$

The region in exon 2 that conferred these effects was subsequently mapped to a 14 amino acid sequence in the $\mathrm{C}$-terminus of the protein. CtBPs were identified as the cellular proteins that interact with a PxDLS peptide consensus within this sequence. ${ }^{8-10}$ Further viral oncoproteins, EBNA3A and EBNA3C, also contain variants of this sequence and bind CtBPs, and EBNA3C requires CtBP-binding for co-transformation with mutant ras. ${ }^{11,12}$ Reports identifying the presence of a functional CtBP-binding consensus sequence in the Drosophila transcriptional repressors, Hairy, Knirps and Snail, ${ }^{13-15}$ were the first of many that have defined a normal cellular function of CtBPs as transcriptional co-repressors of DNA-binding transcription factors. ${ }^{16,17}$ 


\section{CtBP structure and function}

\section{Gene and protein structure}

In Drosophila there is a single $d C t b p$ gene, whereas humans and mice have two CtBP-encoding genes, Ctbp1 and Ctbp2. In humans these map to chromosomes 4p16 and 10q26.13 respectively, the chromosomal location that was initially assigned.to $\mathrm{CtBP} 2{ }^{10}$ having been one of a number of Ctbp pseudogenes. Transcripts from both Ctbp1 and Ctbp2 are widely expressed in both adult tissue, and during development. ${ }^{10,18-20} C t b p 1$ is expressed in a greater number of tissues and often at greater levels than Ctbp2. ${ }^{18,20}$ Interestingly, Ctbp2 expression patterns differ between human and mouse. ${ }^{10}$ In retinal cells, use of an alternative, tissue-specific promoter in the first intron of the Ctbp2 gene leads to the production of mRNA encoding the synaptic ribbon protein RIBEYE, which consists of a large, unique $\mathrm{N}$-terminal domain fused to all but the N-terminal 20 amino acids of CtBP2 ${ }^{21,22}$ Similarly, the protein originally identified as CtBP3/BARS in the rat, ${ }^{23}$ and which is also expressed in human cells, ${ }^{24}$ lacks the first 13 a.a. of CtBP1 and almost certainly represents an alternatively spliced transcript of the Ctbp1 gene. For the purposes of this review, functions so far ascribed solely to CtBP3/BARS will be considered as potential characteristics of all CtBP proteins, whilst the specialised role of RIBEYE will not be discussed further.

The proteins encoded by Ctbp1 and Ctbp 2 share $78 \%$ amino acid identity and $83 \%$ similarity. ${ }^{10}$ They appear to function interchangeably, although evidence is emerging from analysis of mouse models and various post-translational modifications that their activity is differentially regulated. ${ }^{19,25} \mathrm{CtBPs}$ are characterized by a significant degree of homology between their central domain and $\mathrm{NAD}^{+}$-dependent dehydrogenase enzymes (Figure 1). However, whilst CtBPs possess weak in vitro dehydrogenase activity, the significance of this remains unclear. ${ }^{26,27}$ This 
domain does however have an important role as a dimerization site, allowing the formation of homo- and hetero-dimers between CtBP1 and CtBP2. ${ }^{20,26,28}$ Critically, dimerization is stimulated by the occupation of a dinucleotide-binding site within the domain, ${ }^{26,27,29}$ the preferred ligand of which being $\mathrm{NADH}$, which binds to the site with $>100$ fold higher affinity than $\mathrm{NAD}^{+} .30,31$

The three-dimensional structure of CtBPs comprises a single globular domain formed by its $\mathrm{N}$ and C-terminal regions, linked to the core dimerization domain through two flexible hinges. ${ }^{26,28}$ Within this globular domain, the CtBP N-terminus contains a PxDLS peptide binding cleft, and the C-terminus has been proposed to play a regulatory role. ${ }^{28,32}$ Notably, the conformational changes induced upon NADH binding are propagated to the N-terminus, and promote binding to E1A and other PxDLS containing proteins. ${ }^{26,28}$ Importantly, a CtBP dimer has the potential to form the core of a complex containing two PxDLS-containing proteins.

\section{CtBP functions in the nucleus and cytoplasm}

Two distinct functions have been assigned to CtBP proteins - a nuclear role in the regulation of transcription, and a distinct function, thus far only directly ascribed to the CtBP3/BARS isoform, in regulating Golgi membrane maintenance. The role in transcription was first indicated by studies on E1A that showed when exon 1 of E1A is fused to a sequence-specific DNA-binding domain it activated transcription, and this activation is inhibited by the presence, in cis, of the CtBP-binding region of exon $2 .{ }^{33}$ Subsequent fusion of CtBP itself to a DNA-binding domain provided direct evidence that CtBPs repress transcription. ${ }^{15,34,35} \mathrm{~A}$ large number of DNA-binding transcriptional repressors have now been shown to recruit CtBPs, the majority, but not all, ${ }^{36}$ containing a consensus PxDLS sequence ${ }^{16,17,37}$. The best characterized of these are the Snail, 
Slug, and ZEB/ $/ \mathrm{EF} 1$ factors, which together recruit CtBPs to repress the expression of epithelialspecific genes in mesenchymal cells..$^{13,18,38-42}$ These, and other CtBP-recruiting factors with potential roles in the control of apoptosis, are discussed in more detail in subsequent sections.

CtBPs employ a number of mechanisms to regulate gene transcription, as summarized in Figure 2. The most extensively characterized mechanism whereby it acts as a transcriptional corepressor is by the recruitment of a co-repressor complex, which includes class I histone deacetylases (HDACs) and histone methyltransferases (HMTs), and resulting in the co-ordinated modification of chromatin into a repressed state. ${ }^{43} \mathrm{CtBPs}$ can also recruit class II HDACs, ${ }^{44}$ as well as repress transcription without HDAC involvement. ${ }^{35,45}$ An additional mechanism whereby CtBPs appear to act as a co-repressor is by promoting the recruitment of Polycomb proteins to DNA. $^{20,46}$ These repress transcription through several mechanisms, including by compacting chromatin. ${ }^{47}$ Finally, recent research has also determined that CtBPs interact with histone acetyltransferase (HAT) co-activators such as p300, $\mathrm{CBP}$ and $\mathrm{pCAF}$ and prevents their interaction with chromatin. ${ }^{48}$

Independently of its role in regulating transcription, $\mathrm{CtBP}$ (in the form of $\mathrm{CtBP} 3 / \mathrm{BARS}$ ) was cloned as a protein involved in the disassembly of the Golgi complex. ${ }^{23} \mathrm{CtBP} 3 / \mathrm{BARS}$ is able to induce the breakdown of tubular Golgi networks. ${ }^{49}$ It is responsible for driving the fission of Golgi membranes during mitosis and also plays a role in endocytic and exocytic pathways. ${ }^{50-52}$ The distinct nuclear and cytoplasmic functions of CtBPs may be defined by the conformational shift induced by occupation of the dinucleotide binding site, with NADH driving CtBPs towards a nuclear function, and acyl-CoA, which also binds CtBPs, promoting the monomeric, 
cytoplasmic form. ${ }^{28,52}$ Ultimately, it will require the use of specific CtBP mutants to dissect the relative contributions of these distinct functions of CtBPs in cell proliferation and survival.

\section{CtBP functional knockouts reveal key roles for CtBPs in differentiation, cell cycle progression and the suppression of apoptosis}

Before considering the multiplicity of signaling pathways and transcription control networks that CtBPs are involved in, it is useful to review the effects of experimentally abrogating CtBP expression or function on the phenotype of the organism or the individual cell. Homozygous inactivation of the $d C t b p$ gene in Drosophila is lethal, with embryos displaying severe defects in segmentation, consistent with a role for $\mathrm{dCtBP}$ in the function of Hairy, Knirps and Snail transcriptional repressors. ${ }^{13-15}$ In Xenopus embryos, injection of mRNA encoding an xCtBP fusion protein designed to activate, rather than repress, transcription, resulted in similarly dramatic defects such as loss of head and/or eyes and a shortened anterior-posterior axis. ${ }^{53}$ In murine development, homozygous deletion of Ctbp1 results in mice which develop normally, but are smaller than heterozygotes. ${ }^{19} \mathrm{Ctbp} 2^{-/}$embryos exhibited multiple defects, including axial truncations and abnormal heart and brain development, and embryonic lethality occurred due to defects in extra-embryonic vascularization. Crosses between $C t b p 1^{+/-}$and $C t b p 2^{+/-}$revealed further developmental defects, with $C t b p 1^{-/ /} / C t b p 2^{-/-}$embryos showing the most severe phenotype.

Murine embryonic fibroblasts (MEFs) from $C t b p 1^{-/ / C t b p} 2^{-/-}$embryos have been established, and immortalised with SV40 large T antigen. They have defects in CtBP-sensitive gene transcription, 
but normal Golgi apparatus. ${ }^{19}$ They are, however, hypersensitive to apoptosis in response to diverse stimuli including loss of cell-cell contact (anoikis), genotoxic chemotherapeutics, staurosporine, and Fas ligand. ${ }^{54}$ Validated micro-array analysis showed two classes of genes to be constitutively up-regulated in these Ctbp null cells, and suppressed, by CtBP1 re-expression: those encoding epithelial-specific proteins including E-cadherin and several keratins, and proapoptotic proteins including PERP, PTEN, insulin-like growth factor binding proteins, Bax, Noxa and Id-1. ${ }^{54}$ Together with separate evidence that siRNA-mediated CtBP 'knockdown' in human tumor cell lines is sufficient to induce apoptosis in the absence of any additional stress, ${ }^{55}$ these data confirm previous indirect evidence from studies on $\mathrm{E} 1 \mathrm{~A},{ }^{39}$ that $\mathrm{CtBPs}$ function as global repressors of the pro-apoptotic program.

The above studies attributed the effects of CtBP ablation on cellular phenotype to the transcriptional function of CtBPs. Interestingly, another group has independently knocked down CtBP expression in rat cell lines. Rather than apoptosis, they observed $\mathrm{G}_{2}$ arrest, which they demonstrated to be consistent with the disruption of Golgi architecture. ${ }^{50,52}$ Further clarification is undoubtedly warranted.

\section{CtBP regulates multiple transcription factor networks with roles in tumorigenesis and survival}

Whilst the precise mechanisms that link CtBPs to the suppression of pro-apoptotic gene expression programs remain essentially undefined, molecular studies have identified physical and functional involvement of CtBPs with numerous cellular transcription factors. Some of these 
interactions that are likely to have particular relevance to cell survival pathways and tumorigenesis are reviewed in the following sections and summarized in Figure 3.

\section{Repression of the epithelial phenotype}

Negative regulation of the expression of epithelial-specific genes, and particularly those encoding E-cadherin, desmoglein-2, plakoglobin and various keratins, is perhaps the most wellcharacterized function of CtBP proteins. E-cadherin is a calcium-dependent membrane protein required for the formation of adherens junctions, which are essential for cell adhesion and intercellular interactions ${ }^{56}$. It interacts with the actin cytoskeleton via catenins to mediate cellular integrity, polarity and morphogenesis. ${ }^{41,57,58}$ Loss of E-cadherin expression in epithelial cells is associated with an event referred to as epithelial-mesenchymal transformation (EMT). EMT is a major mechanism of vertebrate embryological tissue remodeling, which involves a switch in expression of epithelial- to mesenchymal cell specific genes, resulting in a characteristic motile, migratory mesenchymal phenotype. ${ }^{59}$ EMT and loss of E-cadherin expression is also associated with disease progression, increased malignancy and poor prognosis in many epithelial tumors, including those of the colon, stomach, lung, bladder, oesophagus, prostate and breast. ${ }^{60-66}$ Along with increased invasive properties, one of the key outcomes of EMT is a marked reduction in a cell's susceptibility to pro-apototic stimuli such as loss of cell-cell contact, growth factor withdrawal, and TNF- $\alpha .^{39,67}$

Activation of transcription from the E-cadherin promoter is driven by ubiquitously expressed, and constitutively active transcription factors. Its lack of expression in cells of mesenchymal origin is due primarily to the interaction of Snail, Slug and ZEB/ $\delta \mathrm{EF} 1$ transcriptional repressors 
to E-box elements within their promoters. ${ }^{41,68}$ These repressors all contain PxDLS motifs which are required, at least in part, for their repressor activity. ${ }^{15,18,42,69}$ Their expression and activity is regulated throughout development, and is important during carcinogenesis. Snail expression, for example, is predictive of poor prognosis in breast and hepatocellular carcinoma. ${ }^{70-73} \mathrm{CtBPs}$ can associate with the E-cadherin promoter in cells with a non-epithelial phenotype, ${ }^{43}$ and downregulation of CtBP activity, either by the C-terminus of E1A, or Ctbp gene disruption, results in changes in histone modifications at the E-cadherin promoter, and de-repression of E-cadherin transcription. ${ }^{39,43,54}$ Interestingly, the protein pinin/DRS, which is known to promote the epithelial phenotype, binds CtBPs and relieves its repression of the E-cadherin promoter. ${ }^{74}$

One model that has been proposed, but not yet experimentally tested, is that the repression of pro-apoptotic genes by CtBPs may be an indirect consequence of EMT. Another factor to consider is the recruitment of CtBPs by Snail, Slug or ZEB/SEF1 to the promoters of other genes, such as BRCA2. ${ }^{42}$ It will be of interest to establish whether CtBPs directly associate with the promoters of pro-apoptotic genes, as well as determining the effects of inhibiting CtBP expression in epithelial cells where these repressors are not expressed.

\section{Wnt signaling}

Some of the developemental defects observed following loss of CtBP function are suggestive of defects in the Wnt signaling pathway. ${ }^{53}$ Binding of Wnt to its cell surface receptors results in the accumulation of free cytoplasmic $\beta$-catenin followed by its translocation to the nucleus, where it acts as a co-activator for TCF family transcription factors. ${ }^{75}$ Constitutive activation of this pathway occurs in multiple human cancers, notably colorectal carcinoma where it is associated 
with suppression of apoptosis. ${ }^{76}$ In Xenopus, $\mathrm{xCtBP}$ was found to bind, and act as a co-repressor for xTCF-3, which functions as a transcriptional repressor in the absence of Wnt pathway activation. ${ }^{53}$ In mammalian cells, CtBPs do not interact with TCFs, but rather bind the product of the Adenomatous polyposis coli (APC) gene. ${ }^{77}$ This results in CtBP-mediated suppression of Wnt signaling by sequestering complexes of APC and $\beta$-catenin away from TCF transcription factors. In this context, therefore, CtBPs actually appear to play a role in promoting apoptosis and tumor suppression.

\section{Transforming Growth Factor- $\beta$ (TGF- $\beta$ ) signaling pathways}

A common chromosomal translocation of a number of myeloid malignancies, including acute myeloid leukaemia, chronic myelogenous leukemia and myelodysplastic syndromes, results in the production of a fusion protein with AML1 and Evi-1, driven by the AML1 promoter. ${ }^{78}$ Under normal conditions, Evi-1 is barely detectable in bone marrow and peripheral blood; however over-expression of AML1/Evi-1 is associated with transformation of haematopoietic stem cells. Evi-1 is a transcriptional repressor, and a negative regulator of (TGF- $\beta$ ) signaling. Evi-1 acts on TGF- $\beta$-mediated regulation of cellular proliferation and differentiation by repressing the TGF- $\beta$ activated transcription factor, Smad3. Effective inhibition of TGF- $\beta$ signaling and promotion of cellular transformation by both AML1/Evi-1 and Evi-1, is dependent upon their recruitment of CtBPs, through a PxDLS motif. ${ }^{78-82}$

TGF- $\beta$ has a complex role in regulating apoptotic pathways, and can activate either pro- or antiapoptotic pathways, depending on cell type. ${ }^{83}$ Whilst the interaction with Evi-1 is consistent with an anti-apoptotic role for $\mathrm{CtBPs},{ }^{84} \mathrm{CtBPs}$ also interact with several other key components of 
TGF- $\beta$ signaling pathways that may also control cell survival. TGIF interacts with a TGF- $\beta$ activated Smad complex to repress Smad-mediated transcription, this repression involves the recruitment of CtBPs by a PxDLS motif in TGIF. ${ }^{85}$ The importance of the TGIF-CtBP interaction in human development is demonstrated by an inherited point mutation in the PxDLS motif in TGIF, which results in defects in craniofacial development. ${ }^{85} \mathrm{CtBPs}$ also interact with the inhibitory Smad, Smad6, which contributes to the repression of Id-1 gene transcription ${ }^{86} I d-1$ is up-regulated in $\mathrm{Ctbp}^{-/} \mathrm{Ctbp2^{-/ }} \mathrm{MEFs},{ }^{54}$ and can either induce or suppress apoptosis, depending on the cellular context. ${ }^{87,88} \mathrm{CtBPs}$ are also recruited to Smads indirectly through ZEB/ $/ \mathrm{EF} 1$ proteins, particularly ZEB-2, repressing TGB- $\beta$ induced transcription. ${ }^{89}$

\section{Growth factor receptor, and nuclear hormone receptor signaling}

Ets protein family members are key transcriptional activating factors that transmit signals from growth factor-induced ras-signaling pathways to changes in gene expression, for example through their binding to the serum response element (SRE) on the c-fos promoter. Net is a somewhat atypical Ets family member in that, under certain conditions, it can also function as a

transcriptional repressor. Criqui-Filipe et al ${ }^{32}$ demonstrated that Net contains a CtBP binding site, and in the absence of serum growth factors it recruits CtBPs to actively repress transcription from SRE-dependent promoters.

Ligand-activated nuclear hormone receptors play a key role in regulating cell proliferation, differentiation, and survival in diverse tissue types. Typically, ligand binding to its receptor results in the recruitment of transcriptional co-activators to induce target gene expression. RIP140 is unusual in this respect, as it is recruited to most activated nuclear hormone receptors, 
but acts as a transcriptional repressor. It appears to be essential for suppressing nuclear hormone receptor-induced transcription in certain tissues, to allow key processes such as ovulation and adipogenesis. ${ }^{90}$ Recruitment of CtBPs by RIP140, which is regulated by acetylation of a lysine residue adjacent to the PxDLS sequence on RIP $140,{ }^{36}$ plays an important role in the repressor activity of RIP140..$^{36,91}$

\section{C-terminal binding protein interacting protein (CtIP)}

CtIP was originally identified as a PxDLS-containing binding partner for CtBP. ${ }^{92}$ It is a large, $125 \mathrm{kDa}$ nuclear protein that plays a complex role in transcriptional repression, cell cycle control and DNA damage responses. CtIP acts as a molecular bridge to recruit CtBPs to at least two known transcriptional repressors: the $\mathrm{G}_{1}$ checkpoint regulator, $\mathrm{Rb},{ }^{45}$ and the haemo-lymphoid factor, Ikaros. ${ }^{93}$ In both cases, the CtBP-CtIP complex is thought to co-operate with these factors to co-repress target gene transcription. Mice with heterozygous inactivation of the Ctip gene show increased incidence of multiple tumor types, particularly large lymphomas. This is consistent with a tumor suppressor role for the protein, although the wild-type Ctip allele is not lost in the cancers. ${ }^{94}$ However, homozygous Ctip gene inactivation results in lethality very early in mouse embryogenesis, with cells arresting prior to S phase, and also showing elevated levels of cell death. Experiments on cell lines showed this $\mathrm{G}_{1}$ arrest to be $\mathrm{Rb}$-dependent, and associated with elevated levels of the cyclin dependent kinase inhibitor, p2 $1^{\text {WAF1 }} .{ }^{4}$

Potentially some of these effects of CtIP loss may be accounted for by its interaction with the BRCT domains of the BRCA1 protein. ${ }^{95,96}$ BRCA1 is a well characterized tumor-suppressor protein that plays a central role in DNA damage response. ${ }^{97,98}$ It has been reported that BRCA1 
recruits the CtIP-CtBP complex to repress transcription of BRCA1 target genes such as gadd45 and WAF-1, and that this association is disrupted following DNA damage, ${ }^{99,100}$ although some of these findings have subsequently been disputed. ${ }^{101,102}$ BRCA1-CtIP complex formation is critical for the DNA damage-induced $\mathrm{G}_{2} / \mathrm{M}$ checkpoint, though the role of CtBPs in this is undetermined. ${ }^{103}$

\section{Regulation of p53-dependent pro-apoptotic genes}

P53 is a stress-activated transcription factor that plays a central role in the regulation of cell proliferation, and the induction of apoptosis. ${ }^{104}$ Many of the genes up-regulated in $C t b p 1^{-/ C t b p 2-}$ ${ }^{1}$ MEFs are known transcriptional targets of the pro-apoptotic transcription factor p53, however CtBPs could repress their transcription independently of p53 function. ${ }^{54}$ It seems likely that one consequence of repression of a distinct subset of p53-target genes by CtBPs would be to either dampen, or otherwise modify the cellular response to p53 activation.

CtBPs also directly suppress p53-dependent transcription through its interaction with the Hdm2 oncoprotein. ${ }^{105} \mathrm{Hdm} 2$ is the major cellular regulator of $\mathrm{p} 53$ protein function, inhibiting the interaction of p53 with transcriptional co-activators, and driving its export from the cytoplasm and degradation by the proteosome. ${ }^{106} \mathrm{Hdm} 2$ also recruits CtBPs to $\mathrm{p} 53$, repressing p53dependent promoters such as bax. ${ }^{105}$ This provides an additional mechanism whereby CtBPs suppress apoptosis in cells that retain a functional p53 stress-response pathway. 


\section{Regulation of CtBP function by stress and survival signaling pathways}

In addition to interacting with the known components of signaling pathways described above, it is becoming clear that the activity of CtBPs themselves are regulated by post-translational modification and protein-protein interactions in response to a range of intracellular signals. In some cases, the associated changes in CtBP activity have a direct effect on cell survival.

Before the Ctbp genes were even cloned, their protein products were known to be phosphorylated in a cell-cycle dependent manner, ${ }^{8}$ however the enzymes responsible, and the effect on CtBP function has yet to be clarified. Phosphorylation of CtBP does, however, play a key role in the induction of apoptosis in response to UV-irradiation activated stress-signaling. High doses of UV activate homoedomain interacting protein kinase 2 (HIPK2), an enzyme which has a known role in pro-apoptotic signaling through its phosphorylation and activation of p53. ${ }^{107-}$ ${ }^{109}$ HIPK2 phosphorylates CtBP1 at serine 422 (the site is conserved in CtBP2), which results in the targeting of CtBP1 for ubiquitin-dependent degradation by the proteosome. ${ }^{55,110}$ The subsequent decrease in CtBP protein levels is sufficient to trigger apoptosis in p53 null cells, and either siRNA 'knockdown' of HIPK2, or over-expression of a serine 422 mutant of CtBP1 incapable of being phosphorylated, inhibits UV-induced apoptosis.

CtBPs are also regulated at the level of their subcellular distribution, with nuclear localization being required for transcriptional repressor activity. In most cell types examined, CtBP1 and CtBP2 are predominantly detectable in the nucleus. To some extent, this nuclear localization is a consequence of recruitment by PxDLS-containing transcription factors such as Net. ${ }^{32} \mathrm{CtBP} 1$ is also regulated at this level through two mutually exclusive modifications. SUMOylation at lysine 
428 following interaction with the SUMO E3 ligases Pc2, PIAS1, or PIASx $\beta$ promotes nuclear localization of $\mathrm{CtBP}$, whereas interactions of its C-terminal PDZ domain with neuronal nitric oxide synthase (nNOS), and potentially other proteins, promotes cytoplasmic localization..$^{25,111,112}$ It is not yet clear whether CtBP2 localization and activity is similarly regulated, as the critical SUMOylation consensus site is not conserved, even though CtBP2 is also SUMOylated by Pc2. ${ }^{112}$ Regulation of these interactions in response to cellular signaling has yet to be established. Signaling pathways downstream of growth factor tyrosine kinase receptors do, however, regulate CtBP subcellular localization. The kinase Pak1, which is activated by multiple growth factors including Heregulin and epidermal growth factor, phosphorylates CtBP1 at serine 158. This results in a transient exclusion of CtBP1 from the nucleus following growth factor stimulation. ${ }^{113}$ This serine is conserved in CtBP2, but it is not yet known whether CtBP2 is similarly regulated. This effect is intriguing, given that these factors are associated with cell survival, rather than the induction of apoptosis, and suggest that transient exclusion of CtBPs from the nucleus may be required for certain responses to growth factor stimulation, and does not, in itself, induce apoptosis.

Probably the most interesting mechanism of regulation of CtBPs is the conformational change induced by occupancy of the dinucleotide binding site. NADH binding to CtBPs promotes both its dimerization and interaction with PxDLS-containing transcriptional repressors, and consequently promotes its ability to repress transcription from a set of target promoters. ${ }^{29,31,44}$ Conversely, the NADH unbound form preferentially binds to Hdm2, which represses p53dependent transcription, ${ }^{105}$ and also to $\mathrm{p} 300 / \mathrm{CBP}$, with potentially broad ranging effects on p300-dependent gene transcription. ${ }^{48}$ Levels of intracellular NADH show dramatic changes at 
birth, in response to ethanol, cellular hypoxia, and in metabolic disease such as diabetes. Potentially, CtBPs may regulate broad-ranging changes in gene expression patterns in response to these alterations in cellular metabolism.

\section{Future perspectives}

CtBPs are clearly involved in numerous transcriptional regulatory programs and intracellular signaling pathways that regulate cell proliferation, differentiation and survival. Given that loss of CtBP expression induces either apoptosis or $\mathrm{G}_{2}$ arrest, one might predict that cellular levels of CtBPs might be elevated in diseases of dysregulated proliferation and survival, such as cancer. However the pleiotrophic nature of CtBPs activities may complicate its role in carcinogenesis. Indeed, in one of the few published studies of CtBP protein expression in human primary cancers, CtBP1 levels were markedly reduced in malignant melanomas, compared to primary melanocytes. ${ }^{114}$ This was found to be consistent with a role for CtBPs in suppressing the expression of MIA, a protein associated with malignant progression in this tumor type, ${ }^{114,115}$ and highlights the need for further studies into CtBP expression patterns in other tumor types.

In contrast, microarray analysis of follicular thyroid carcinomas found a mean 3.6 fold increase in Ctbp2 mRNA levels in a defined subset of these tumors. ${ }^{116}$ Unfortunately, the commercial qPCR assay used to validate these findings recognises the unique exon 1 found in the ribeye transcript, and therefore the role of CtBP2 in these tumors remains to be confirmed. CtBPs are, however, ubiquitously expressed, and are detectable in the majority of cancer cell lines that have been examined. The finding that cells derived from $C t b p 1^{-/} C t b p 2^{-/}$embryos proliferate normally, whereas siRNA-mediated knockdown of CtBPs in cancer cell lines can induce 
apoptosis, could have a number of explanations, one of which is that some of genetic changes associated with cellular transformation render cells more dependent on the suppression of proapoptotic genes by CtBPs. This would be entirely consistent with the original observations that full length E1A is required for cellular immortalization, whereas exon 2, which functions at least in part by preventing the interaction of CtBPs with cellular PxDLS containing transcription factors, ${ }^{117}$ suppresses transformation in the presence of mutant ras.

In summary, CtBPs are clearly key regulators of both gene transcription and organelle assembly. They have been implicated in the context of a striking number of transcriptional regulatory networks, and intracellular signaling pathways. They play an essential pro-survival role in tumour-derived cells, though, intriguingly the mechanism basis of this critical function remains to be clarified. Further studies will undoubtedly shed light on these multifaceted molecules.

\section{References}

1. Sang N, Caro J, Giordano A. Adenoviral E1A: everlasting tool, versatile applications, continuous contributions and new hypotheses. Front Biosci 2002; 7: d407-13.

2. Endter C, Dobner T. Cell transformation by human adenoviruses. Curr Top Microbiol Immunol 2004; 273: 163-214.

3. Schneider JF, Fisher F, Goding CR, Jones NC. Mutational analysis of the adenovirus E1a gene: the role of transcriptional regulation in transformation. Embo J 1987; 6: 2053-60.

4. Whyte P, Williamson NM, Harlow E. Cellular targets for transformation by the adenovirus E1A proteins. Cell 1989; 56: 67-75. 
5. Subramanian T, La Regina M, Chinnadurai G. Enhanced ras oncogene mediated cell transformation and tumorigenesis by adenovirus 2 mutants lacking the $\mathrm{C}$-terminal region of E1a protein. Oncogene 1989; 4: 415-20.

6. Subramanian T, Malstrom SE, Chinnadurai G. Requirement of the C-terminal region of adenovirus E1a for cell transformation in cooperation with E1b. Oncogene 1991; 6: 1171-3.

7. Chinnadurai G. Modulation of oncogenic transformation by the human adenovirus E1A C-terminal region. Curr Top Microbiol Immunol 2004; 273: 139-61.

8. Boyd JM, Subramanian T, Schaeper U et al. A region in the C-terminus of adenovirus $2 / 5$ E1a protein is required for association with a cellular phosphoprotein and important for the negative modulation of T24-ras mediated transformation, tumorigenesis and metastasis. Embo J 1993; 12: 469-78.

9. Schaeper U, Boyd JM, Verma S et al. Molecular cloning and characterization of a cellular phosphoprotein that interacts with a conserved C-terminal domain of adenovirus E1A involved in negative modulation of oncogenic transformation. Proc Natl Acad Sci U S A 1995; 92: 10467-71.

10. Katsanis N, Fisher EM. A novel C-terminal binding protein (CTBP2) is closely related to CTBP1, an adenovirus E1A-binding protein, and maps to human chromosome 21q21.3. Genomics 1998; 47: 294-9.

11. Touitou R, Hickabottom M, Parker G et al. Physical and functional interactions between the corepressor CtBP and the Epstein-Barr virus nuclear antigen EBNA3C. J Virol 2001; 75: 7749-55. 
12. Hickabottom M, Parker GA, Freemont $\mathrm{P}$ et al. Two nonconsensus sites in the EpsteinBarr virus oncoprotein EBNA3A cooperate to bind the co-repressor carboxyl-terminalbinding protein (CtBP). J Biol Chem 2002; 277: 47197-204.

13. Nibu Y, Zhang H, Bajor E et al. dCtBP mediates transcriptional repression by Knirps, Kruppel and Snail in the Drosophila embryo. Embo J 1998; 17: 7009-20.

14. Poortinga G, Watanabe M, Parkhurst SM. Drosophila CtBP: a Hairy-interacting protein required for embryonic segmentation and hairy-mediated transcriptional repression. Embo J 1998; 17: 2067-78.

15. Nibu Y, Zhang H, Levine M. Interaction of short-range repressors with Drosophila CtBP in the embryo. Science 1998; 280: 101-4.

16. Chinnadurai G. CtBP, an unconventional transcriptional corepressor in development and oncogenesis. Mol Cell 2002; 9: 213-24.

17. Turner J, Crossley M. The CtBP family: enigmatic and enzymatic transcriptional corepressors. Bioessays 2001; 23: 683-90.

18. Furusawa T, Moribe H, Kondoh H, Higashi Y. Identification of CtBP1 and CtBP2 as corepressors of zinc finger-homeodomain factor deltaEF1. Mol Cell Biol 1999; 19: 858190.

19. Hildebrand JD, Soriano P. Overlapping and unique roles for C-terminal binding protein 1 (CtBP1) and CtBP2 during mouse development. Mol Cell Biol 2002; 22: 5296-307.

20. Sewalt RG, Gunster MJ, van der Vlag J et al. C-Terminal binding protein is a transcriptional repressor that interacts with a specific class of vertebrate Polycomb proteins. Mol Cell Biol 1999; 19: 777-87. 
21. Schmitz F, Konigstorfer A, Sudhof TC. RIBEYE, a component of synaptic ribbons: a protein's journey through evolution provides insight into synaptic ribbon function. Neuron 2000; 28: 857-72.

22. Piatigorsky J. Dual use of the transcriptional repressor (CtBP2)/ribbon synapse (RIBEYE) gene: how prevalent are multifunctional genes? Trends Neurosci 2001; 24: $555-7$.

23. Spano S, Silletta MG, Colanzi A et al. Molecular cloning and functional characterization of brefeldin A-ADP-ribosylated substrate. A novel protein involved in the maintenance of the Golgi structure. J Biol Chem 1999; 274: 17705-10.

24. Monleon I, Iturralde M, Martinez-Lorenzo MJ et al. Lack of Fas/CD95 surface expression in highly proliferative leukemic cell lines correlates with loss of CtBP/BARS and redirection of the protein toward giant lysosomal structures. Cell Growth Differ 2002; 13: 315-24.

25. Lin X, Sun B, Liang M et al. Opposed regulation of corepressor CtBP by SUMOylation and PDZ binding. Mol Cell 2003; 11: 1389-96.

26. Kumar V, Carlson JE, Ohgi KA et al. Transcription corepressor CtBP is an $\mathrm{NAD}(+)-$ regulated dehydrogenase. Mol Cell 2002; 10: 857-69.

27. Balasubramanian P, Zhao LJ, Chinnadurai G. Nicotinamide adenine dinucleotide stimulates oligomerization, interaction with adenovirus E1A and an intrinsic dehydrogenase activity of CtBP. FEBS Lett 2003; 537: 157-60.

28. Nardini M, Spano S, Cericola C et al. CtBP/BARS: a dual-function protein involved in transcription co-repression and Golgi membrane fission. Embo J 2003; 22: 3122-30. 
29. Thio SS, Bonventre JV, Hsu SI. The CtBP2 co-repressor is regulated by NADHdependent dimerization and possesses a novel N-terminal repression domain. Nucleic Acids Res 2004; 32: 1836-47.

30. Zhang Q, Piston DW, Goodman RH. Regulation of corepressor function by nuclear NADH. Science 2002; 295: 1895-7.

31. Fjeld CC, Birdsong WT, Goodman RH. Differential binding of NAD+ and NADH allows the transcriptional corepressor carboxyl-terminal binding protein to serve as a metabolic sensor. Proc Natl Acad Sci U S A 2003; 100: 9202-7.

32. Criqui-Filipe P, Ducret C, Maira SM, Wasylyk B. Net, a negative Ras-switchable TCF, contains a second inhibition domain, the CID, that mediates repression through interactions with CtBP and de-acetylation. Embo J 1999; 18: 3392-403.

33. Sollerbrant K, Chinnadurai G, Svensson C. The CtBP binding domain in the adenovirus E1A protein controls CR1-dependent transactivation. Nucleic Acids Res 1996; 24: 257884.

34. Turner J, Crossley M. Cloning and characterization of mCtBP2, a co-repressor that associates with basic Kruppel-like factor and other mammalian transcriptional regulators. Embo J 1998; 17: 5129-40.

35. Koipally J, Georgopoulos K. Ikaros interactions with CtBP reveal a repression mechanism that is independent of histone deacetylase activity. J Biol Chem 2000; 275 : 19594-602.

36. Vo N, Fjeld C, Goodman RH. Acetylation of nuclear hormone receptor-interacting protein RIP140 regulates binding of the transcriptional corepressor CtBP. Mol Cell Biol 2001; 21: 6181-8. 
37. Molloy DP, Barral PM, Bremner KH et al. Structural determinants outside the PXDLS sequence affect the interaction of adenovirus E1 A, C-terminal interacting protein and Drosophila repressors with C-terminal binding protein. Biochim Biophys Acta 2001; 1546: 55-70.

38. Postigo AA, Dean DC. ZEB represses transcription through interaction with the corepressor CtBP. Proc Natl Acad Sci U S A 1999; 96: 6683-8.

39. Grooteclaes ML, Frisch SM. Evidence for a function of CtBP in epithelial gene regulation and anoikis. Oncogene 2000; 19: 3823-8.

40. Frisch SM. Tumor suppression activity of adenovirus E1a protein: anoikis and the epithelial phenotype. Adv Cancer Res 2001; 80: 39-49.

41. Peinado H, Portillo F, Cano A. Transcriptional regulation of cadherins during development and carcinogenesis. Int J Dev Biol 2004; 48: 365-75.

42. Tripathi MK, Misra S, Khedkar SV et al. Regulation of BRCA2 Gene Expression by the SLUG Repressor Protein in Human Breast Cells. J Biol Chem 2005; 280: 17163-71.

43. Shi Y, Sawada J, Sui G et al. Coordinated histone modifications mediated by a CtBP corepressor complex. Nature 2003; 422: 735-8.

44. Zhang CL, McKinsey TA, Lu JR, Olson EN. Association of COOH-terminal-binding protein (CtBP) and MEF2-interacting transcription repressor (MITR) contributes to transcriptional repression of the MEF2 transcription factor. J Biol Chem 2001; 276: 35-9.

45. Meloni AR, Smith EJ, Nevins JR. A mechanism for Rb/p130-mediated transcription repression involving recruitment of the CtBP corepressor. Proc Natl Acad Sci U S A 1999; 96: 9574-9. 
46. Srinivasan L, Atchison ML. YY1 DNA binding and PcG recruitment requires CtBP. Genes Dev 2004; 18: 2596-601.

47. Levine SS, King IF, Kingston RE. Division of labor in polycomb group repression. Trends Biochem Sci 2004; 29: 478-85.

48. Kim JH, Cho EJ, Kim ST, Youn HD. CtBP represses p300-mediated transcriptional activation by direct association with its bromodomain. Nat Struct Mol Biol 2005.

49. Weigert R, Silletta MG, Spano $\mathrm{S}$ et al. CtBP/BARS induces fission of Golgi membranes by acylating lysophosphatidic acid. Nature 1999; 402: 429-33.

50. Hidalgo Carcedo C, Bonazzi M, Spano S et al. Mitotic Golgi partitioning is driven by the membrane-fissioning protein CtBP3/BARS. Science 2004; 305: 93-6.

51. Diao A, Lowe M. Cell biology. The Golgi goes fission. Science 2004; 305: 48-9.

52. Bonazzi M, Spano S, Turacchio G et al. CtBP3/BARS drives membrane fission in dynamin-independent transport pathways. Nat Cell Biol 2005.

53. Brannon M, Brown JD, Bates R et al. XCtBP is a XTcf-3 co-repressor with roles throughout Xenopus development. Development 1999; 126: 3159-70.

54. Grooteclaes M, Deveraux Q, Hildebrand J et al. C-terminal-binding protein corepresses epithelial and proapoptotic gene expression programs. Proc Natl Acad Sci U S A 2003; 100: 4568-73.

55. Zhang Q, Yoshimatsu Y, Hildebrand J et al. Homeodomain interacting protein kinase 2 promotes apoptosis by downregulating the transcriptional corepressor CtBP. Cell 2003; 115: $177-86$.

56. Frisch SM. E1a induces the expression of epithelial characteristics. J Cell Biol 1994; 127: 1085-96. 
57. Gooding JM, Yap KL, Ikura M. The cadherin-catenin complex as a focal point of cell adhesion and signalling: new insights from three-dimensional structures. Bioessays 2004; 26: 497-511.

58. Patel SD, Chen CP, Bahna F et al. Cadherin-mediated cell-cell adhesion: sticking together as a family. Curr Opin Struct Biol 2003; 13: 690-8.

59. Hay ED. The mesenchymal cell, its role in the embryo, and the remarkable signaling mechanisms that create it. Dev Dyn 2005; 233: 706-20.

60. Wheeler JM. Epigenetics, mismatch repair genes and colorectal cancer. Ann R Coll Surg Engl 2005; 87: 15-20.

61. Wang HD, Ren J, Zhang L. CDH1 germline mutation in hereditary gastric carcinoma. World J Gastroenterol 2004; 10: 3088-93.

62. Chen XF, Zhang HT, Qi QY et al. Expression of E-cadherin and nm23 is associated with the clinicopathological factors of human non-small cell lung cancer in China. Lung Cancer 2005; 48: 69-76.

63. Syrigos KN, Karapanagiotou E, Harrington KJ. The clinical significance of molecular markers to bladder cancer. Hybrid Hybridomics 2004; 23: 335-42.

64. Nair KS, Naidoo R, Chetty R. Expression of cell adhesion molecules in oesophageal carcinoma and its prognostic value. J Clin Pathol 2005; 58: 343-51.

65. Quinn DI, Henshall SM, Sutherland RL. Molecular markers of prostate cancer outcome. Eur J Cancer 2005; 41: 858-87.

66. Simpson PT, Reis-Filho JS, Gale T, Lakhani SR. Molecular evolution of breast cancer. J Pathol 2005; 205: 248-54. 
67. Vega S, Morales AV, Ocana $\mathrm{OH}$ et al. Snail blocks the cell cycle and confers resistance to cell death. Genes Dev 2004; 18: 1131-43.

68. Hennig G, Behrens J, Truss M et al. Progression of carcinoma cells is associated with alterations in chromatin structure and factor binding at the E-cadherin promoter in vivo. Oncogene 1995; 11: 475-84.

69. Hemavathy $\mathrm{K}, \mathrm{Hu} \mathrm{X}$, Ashraf SI et al. The repressor function of snail is required for Drosophila gastrulation and is not replaceable by Escargot or Worniu. Dev Biol 2004; 269: 411-20.

70. Sugimachi K, Tanaka S, Kameyama $\mathrm{T}$ et al. Transcriptional repressor snail and progression of human hepatocellular carcinoma. Clin Cancer Res 2003; 9: 2657-64.

71. Blanco MJ, Moreno-Bueno G, Sarrio D et al. Correlation of Snail expression with histological grade and lymph node status in breast carcinomas. Oncogene 2002; 21: 3241-6.

72. Elloul S, Elstrand MB, Nesland JM et al. Snail, Slug, and Smad-interacting protein 1 as novel parameters of disease aggressiveness in metastatic ovarian and breast carcinoma. Cancer 2005; 103: 1631-43.

73. Moody SE, Perez D, Pan TC et al. The transcriptional repressor Snail promotes mammary tumor recurrence. Cancer Cell 2005; 8: 197-209.

74. Alpatov R, Munguba GC, Caton P et al. Nuclear speckle-associated protein Pnn/DRS binds to the transcriptional corepressor $\mathrm{CtBP}$ and relieves CtBP-mediated repression of the E-cadherin gene. Mol Cell Biol 2004; 24: 10223-35.

75. Doucas H, Garcea G, Neal CP et al. Changes in the Wnt signalling pathway in gastrointestinal cancers and their prognostic significance. Eur J Cancer 2005; 41: 365-79. 
76. Chen T, Yang I, Irby R et al. Regulation of caspase expression and apoptosis by adenomatous polyposis coli. Cancer Res 2003; 63: 4368-74.

77. Hamada F, Bienz M. The APC tumor suppressor binds to C-terminal binding protein to divert nuclear beta-catenin from TCF. Dev Cell 2004; 7: 677-85.

78. Sood R, Talwar-Trikha A, Chakrabarti SR, Nucifora G. MDS1/EVI1 enhances TGFbeta1 signaling and strengthens its growth-inhibitory effect but the leukemia-associated fusion protein AML1/MDS1/EVI1, product of the $\mathrm{t}(3 ; 21)$, abrogates growth-inhibition in response to TGF-beta1. Leukemia 1999; 13: 348-57.

79. Hirai H, Izutsu K, Kurokawa M, Mitani K. Oncogenic mechanisms of Evi-1 protein. Cancer Chemother Pharmacol 2001; 48 Suppl 1: S35-40.

80. Izutsu K, Kurokawa M, Imai Y et al. The corepressor CtBP interacts with Evi-1 to repress transforming growth factor beta signaling. Blood 2001; 97: 2815-22.

81. Palmer S, Brouillet JP, Kilbey A et al. Evi-1 transforming and repressor activities are mediated by CtBP co-repressor proteins. J Biol Chem 2001; 276: 25834-40.

82. Alliston T, Ko TC, Cao Y et al. Repression of BMP and activin-inducible transcription by Evi-1. J Biol Chem 2005.

83. Siegel PM, Massague J. Cytostatic and apoptotic actions of TGF-beta in homeostasis and cancer. Nat Rev Cancer 2003; 3: 807-21.

84. Mitani K. Molecular mechanisms of leukemogenesis by AML1/EVI-1. Oncogene 2004; 23: 4263-9.

85. Melhuish TA, Wotton D. The interaction of the carboxyl terminus-binding protein with the Smad corepressor TGIF is disrupted by a holoprosencephaly mutation in TGIF. J Biol Chem 2000; 275: 39762-6. 
86. Lin X, Liang YY, Sun B et al. Smad6 recruits transcription corepressor CtBP to repress bone morphogenetic protein-induced transcription. Mol Cell Biol 2003; 23: 9081-93.

87. Parrinello S, Lin CQ, Murata K et al. Id-1, ITF-2, and Id-2 comprise a network of helixloop-helix proteins that regulate mammary epithelial cell proliferation, differentiation, and apoptosis. J Biol Chem 2001; 276: 39213-9.

88. Wong YC, Wang X, Ling MT. Id-1 expression and cell survival. Apoptosis 2004; 9: 27989.

89. Postigo AA, Depp JL, Taylor JJ, Kroll KL. Regulation of Smad signaling through a differential recruitment of coactivators and corepressors by ZEB proteins. Embo J 2003; 22: $2453-62$.

90. Steel JH, White R, Parker MG. Role of the RIP140 corepressor in ovulation and adipose biology. J Endocrinol 2005; 185: 1-9.

91. Castet A, Boulahtouf A, Versini G et al. Multiple domains of the Receptor-Interacting Protein 140 contribute to transcription inhibition. Nucleic Acids Res 2004; 32: 1957-66.

92. Schaeper U, Subramanian T, Lim L et al. Interaction between a cellular protein that binds to the $\mathrm{C}$-terminal region of adenovirus $\mathrm{E} 1 \mathrm{~A}(\mathrm{CtBP})$ and a novel cellular protein is disrupted by E1A through a conserved PLDLS motif. J Biol Chem 1998; 273: 8549-52.

93. Koipally J, Georgopoulos K. Ikaros-CtIP interactions do not require C-terminal binding protein and participate in a deacetylase-independent mode of repression. J Biol Chem 2002; 277: 23143-9.

94. Chen PL, Liu F, Cai S et al. Inactivation of CtIP Leads to Early Embryonic Lethality Mediated by G1 Restraint and to Tumorigenesis by Haploid Insufficiency. Mol Cell Biol 2005; $25: 3535-42$. 
95. Wong AK, Ormonde PA, Pero R et al. Characterization of a carboxy-terminal BRCA1 interacting protein. Oncogene 1998; 17: 2279-85.

96. Yu X, Wu LC, Bowcock AM et al. The C-terminal (BRCT) domains of BRCA1 interact in vivo with CtIP, a protein implicated in the CtBP pathway of transcriptional repression. J Biol Chem 1998; 273: 25388-92.

97. Yoshida K, Miki Y. Role of BRCA1 and BRCA2 as regulators of DNA repair, transcription, and cell cycle in response to DNA damage. Cancer Sci 2004; 95: 866-71.

98. Powell SN, Kachnic LA. Roles of BRCA1 and BRCA2 in homologous recombination, DNA replication fidelity and the cellular response to ionizing radiation. Oncogene 2003; 22: 5784-91.

99. Li S, Chen PL, Subramanian T et al. Binding of CtIP to the BRCT repeats of BRCA1 involved in the transcription regulation of $\mathrm{p} 21$ is disrupted upon DNA damage. J Biol Chem 1999; 274: 11334-8.

100. Li S, Ting NS, Zheng L et al. Functional link of BRCA1 and ataxia telangiectasia gene product in DNA damage response. Nature 2000; 406: 210-5.

101. Yu X, Baer R. Nuclear localization and cell cycle-specific expression of CtIP, a protein that associates with the BRCA1 tumor suppressor. J Biol Chem 2000; 275: 18541-9.

102. Wu-Baer F, Baer R. Effect of DNA damage on a BRCA1 complex. Nature 2001; 414: 36.

103. Yu X, Chen J. DNA damage-induced cell cycle checkpoint control requires CtIP, a phosphorylation-dependent binding partner of BRCA1 C-terminal domains. Mol Cell Biol 2004; 24: 9478-86.

104. Vogelstein B, Lane D, Levine AJ. Surfing the p53 network. Nature 2000; 408: 307-10. 
105. Mirnezami AH, Campbell SJ, Darley M et al. Hdm2 recruits a hypoxia-sensitive corepressor to negatively regulate p53-dependent transcription. Curr Biol 2003; 13: 12349.

106. Momand J, Wu HH, Dasgupta G. MDM2--master regulator of the p53 tumor suppressor protein. Gene 2000; 242: 15-29.

107. Kim YH, Choi CY, Lee SJ et al. Homeodomain-interacting protein kinases, a novel family of co-repressors for homeodomain transcription factors. J Biol Chem 1998; 273: 25875-9.

108. D'Orazi G, Cecchinelli B, Bruno T et al. Homeodomain-interacting protein kinase-2 phosphorylates p53 at Ser 46 and mediates apoptosis. Nat Cell Biol 2002; 4: 11-9.

109. Hofmann TG, Moller A, Sirma H et al. Regulation of p53 activity by its interaction with homeodomain-interacting protein kinase-2. Nat Cell Biol 2002; 4: 1-10.

110. Zhang Q, Nottke A, Goodman RH. Homeodomain-interacting protein kinase-2 mediates CtBP phosphorylation and degradation in UV-triggered apoptosis. Proc Natl Acad Sci U S A 2005; 102: 2802-7.

111. Riefler GM, Firestein BL. Binding of neuronal nitric-oxide synthase (nNOS) to carboxylterminal-binding protein ( $\mathrm{CtBP}$ ) changes the localization of $\mathrm{CtBP}$ from the nucleus to the cytosol: a novel function for targeting by the PDZ domain of nNOS. J Biol Chem 2001; 276: $48262-8$.

112. Kagey MH, Melhuish TA, Wotton D. The polycomb protein Pc2 is a SUMO E3. Cell 2003; 113: 127-37.

113. Barnes CJ, Vadlamudi RK, Mishra SK et al. Functional inactivation of a transcriptional corepressor by a signaling kinase. Nat Struct Biol 2003; 10: 622-8. 
114. Poser I, Golob M, Weidner M et al. Down-regulation of COOH-terminal binding protein expression in malignant melanomas leads to induction of MIA expression. Cancer Res 2002; 62: 5962-6.

115. Poser I, Bosserhoff AK. Transcription factors involved in development and progression of malignant melanoma. Histol Histopathol 2004; 19: 173-88.

116. Lui WO, Foukakis T, Liden J et al. Expression profiling reveals a distinct transcription signature in follicular thyroid carcinomas with a PAX8-PPAR(gamma) fusion oncogene. Oncogene 2005; 24: 1467-76.

117. Johansson C, Zhao H, Bajak E et al. Impact of the interaction between adenovirus E1A and CtBP on host cell gene expression. Virus Res 2005. 
Fig 1

Dimerization
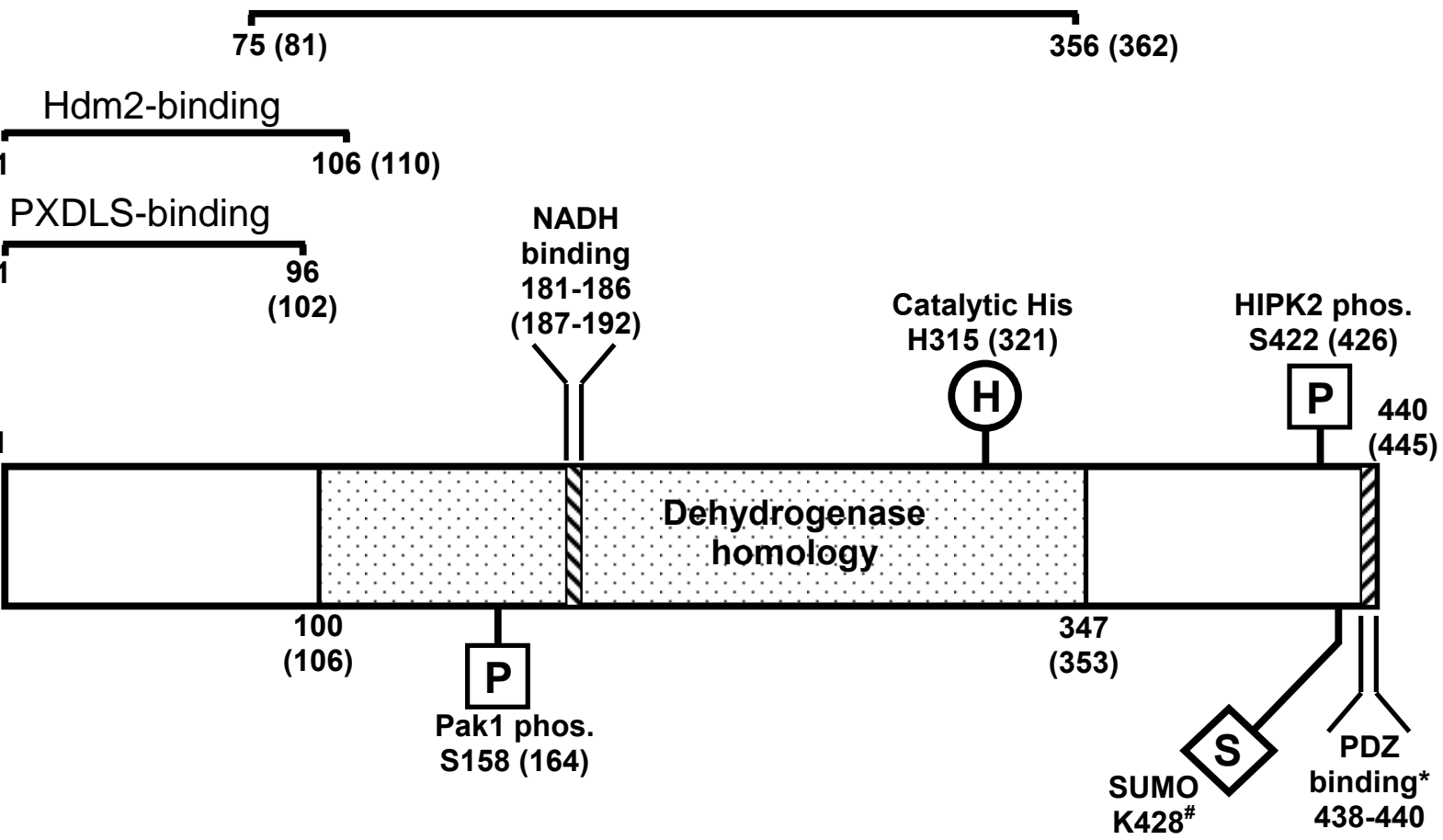

Fig 2 

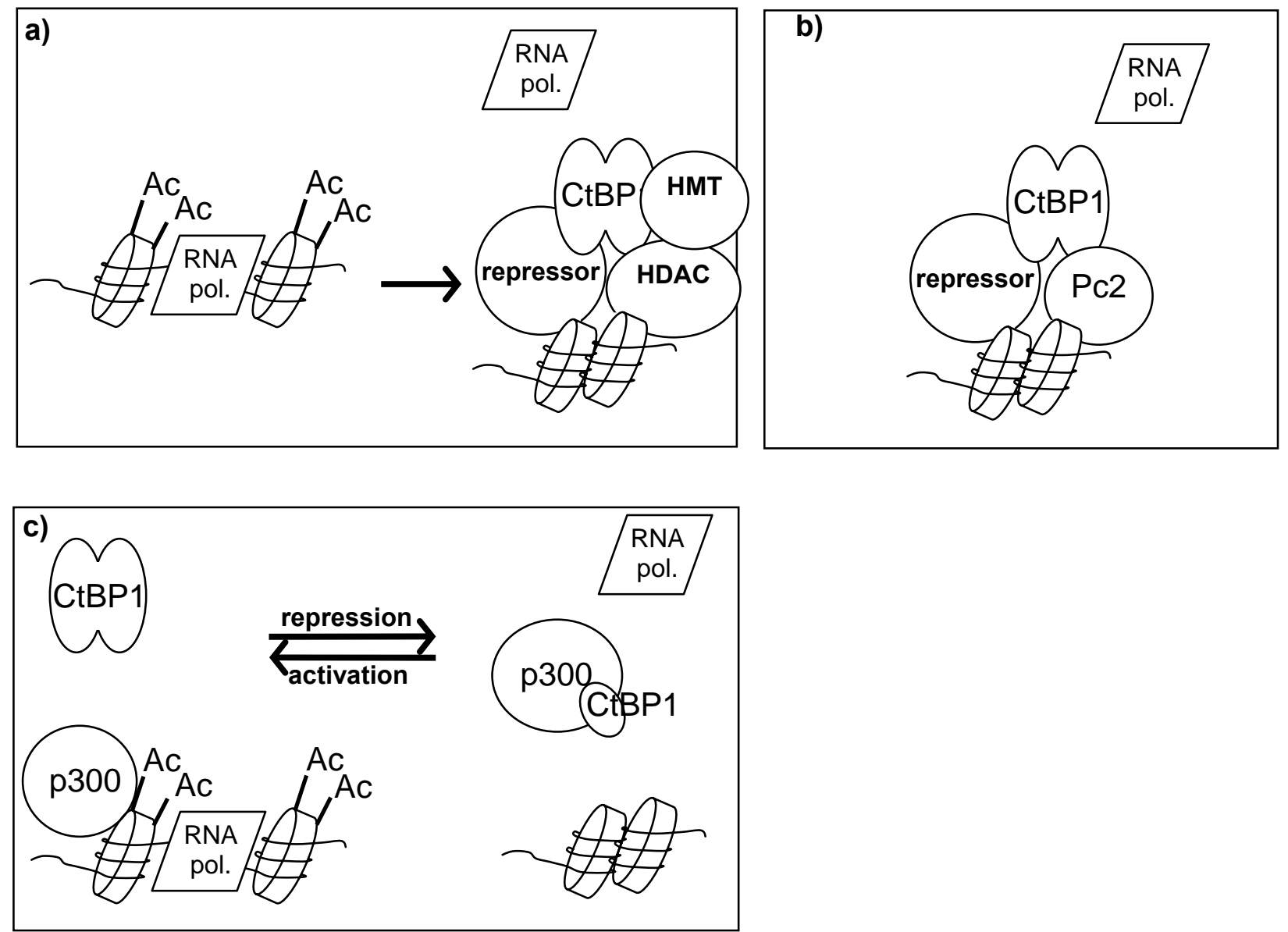
Fig 3

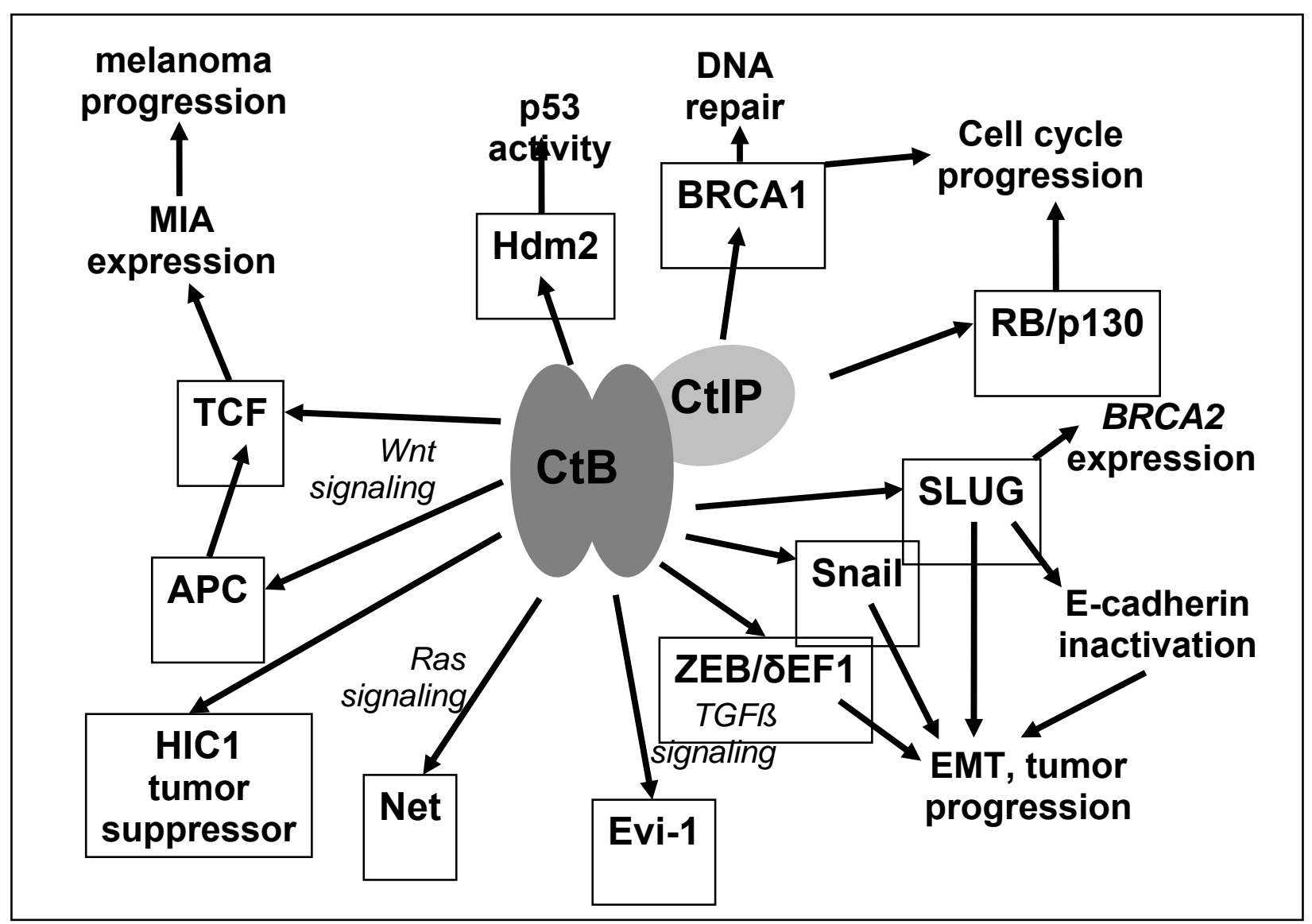

